\title{
Fonoaudiología y estética facial: experiencia de Brasil en la Atención Primaria de Salud
}

\section{Speech Therapy and Facial Esthetic: Experience of Brazil in Primary Health Care}

Axel Pavez R.

Fonoaudiólogo

Universidad de Chile, Chile

Taysa M. Silva

Fonoaudióloga

FEAD-Minas Gerais

Pontificia Universidade Católica de

Minas Gerais, Brasil
Contacto con el autor: Axel Pavez R. Exequiel Fernández 90, Ñuñoa

Santiago - Chile Tel: (569) 93117347 Correo-e: a_pavez@ug.uchile.cl

Recibido: $13 / 07 / 2015$ Aceptado: 28/08/2015

\section{RESUMEN}

La fonoaudiología es una disciplina relativamente nueva en Chile por lo cual se encuentra en pleno proceso de crecimiento y desarrollo. En este contexto, en el área de Motricidad Orofacial ha surgido hace algunos años una subespecialidad denominada "estética facial" que aborda la musculatura facial y sus alteraciones, ya sean secundarias al proceso natural de envejecimiento $\mathrm{y} / \mathrm{o}$ a factores externos. Brasil, país pionero en esta práctica, ha desarrollado diversos planes de intervención en salud. A través de la creación de Núcleos de Apoyo a la Salud de la Familia, se ha incluido la estética facial en el contexto de atención primaria de salud. Es así que en la ciudad de Sete Lagoas, Minas Gerais se ha desarrollado un programa de estética facial con el objetivo de fomentar la integración del cuidado físico y mental de los usuarios. La evidencia científica muestra los resultados de la fonoaudiología estética, relajando la musculatura facial, disminuyendo arrugas y líneas de expresión y mejorando la sensación de bienestar de las personas. Se plantea la importancia de ampliar el conocimiento del profesional fonoaudiólogo chileno y desarrollar nuevas áreas de desempeño para contribuir en el beneficio de la población.

Palabras clave: fonoaudiología, motricidad orofacial, estética facial, fonoaudiología estética, atención primaria de salud.

\begin{abstract}
Speech Therapy is relatively new discipline in Chile, therefore, it is in the process of growth and development. As part of the orofacial motricity area, a new specialty has began to develop: Facial esthetic. This new area deals with facial muscles and their alterations caused by natural aging processes and/or external factors. Brazil, a pioneer country in this practice, has developed several health intervention plans. In this respect, facial esthetic has been included in the context of primary health care, through the creation of Family Health Support Centers. For instance, a program of facial esthetic has been implemented in the city of Sete Lagoas, Minas Gerais. This program aims to promote the integration of physical and mental care. Scientific evidence shows that esthetic's speech therapy produce relaxation of facial muscles, reducing wrinkles and fine lines and improving the people's sense of well-being. Therefore, increasing the knowledge about this area in Chilean speech therapists and developing new areas of professional performance would be important issues to address.
\end{abstract}

Keywords: speech theraphy, orofacial motricity, facial esthetic, esthetic speech theraphy, primary health care. 


\section{Introducción}

La fonoaudiología es una disciplina relativamente nueva en Chile, por lo cual se encuentra en pleno proceso de crecimiento y desarrollo. En este contexto durante los últimos años se ha visto una amplia evolución en el campo de acción del fonoaudiólogo(a) en el área de Motricidad Orofacial (MO). Esta área está orientada al estudio, investigación, prevención, evaluación, diagnóstico y rehabilitación de los aspectos estructurarles y funcionales de las regiones orofaciales y cervicales (Comitê de Motricidade Orofacial, 2004).

En este marco, Brasil ha sido uno de los países pioneros en potenciar el área y contribuir en forma considerable a su desarrollo. Es así que se han impulsado diversos campos de acción en motricidad orofacial, uno de los cuales es la estética facial desarrollada desde 1998 (Franco, 2009). Según lo establecido por el Consejo Federal Brasilero de Fonoaudiología dicha motricidad tiene por objetivo evaluar, prevenir y equilibrar la musculatura facial y cervical, buscando la simetría y armonía de las estructuras involucradas en el movimiento orofacial, con el fin de favorecer la estética facial (Conselho Federal de Fonoaudiologia, 2008). Se incluyen aquí las alteraciones secundarias al proceso natural de envejecimiento y/o factores externos, por ejemplo, quemaduras o parálisis facial. Por su parte Magda Franco, creadora del conocido método "MZ Estética Facial", acota la estética a la reducción de los efectos del proceso de envejecimiento y propone en su libro que la fonoaudiología estética tiene por objetivo la suavización de las líneas de expresión, arrugas y envejecimiento de la cara, a través del reequilibrio de la musculatura orofacial y de las funciones estomatognáticas (Franco, 2009).

La acción fonoaudiológica en estética aún es un tema reciente, por lo tanto el interés en esta temática aumenta cada día (Souza, Morais, Silva y Cunha, 2005). Se sabe que la actividad de los músculos faciales asociada a movimientos exagerados de la mímica facial y/o alteraciones miofuncionales, orofaciales y cervicales provocan marcas de expresión en el rostro y disminuyen su tonicidad y elasticidad; tales cambios se incrementan con el proceso de envejecimiento (Franco, 2009; Mattia, Czlusniak y Ricci, 2008).

El trabajo en estética facial busca lograr sus objetivos mediante la relajación de la musculatura orofacial y suavización de las líneas de expresión a través de masajes, movimientos específicos de la musculatura orofacial, disminución en el abuso de la mímica facial, reorganización postural, promoción de la suavización de la fisonomía, entre otros (Franco, 2004; Mattia et al., 2008). Además de ayudar a las personas en la búsqueda del rejuvenecimiento mediante las técnicas mencionadas, el fonoaudiólogo cumple un rol compartido con el usuario, creando conciencia de los diferentes movimientos y patrones adecuados para lograr que los cambios inherentes al paso del tiempo sean suaves y naturales. De esta forma, la intervención es indicada para personas que buscan métodos de rejuvenecimiento naturales y no invasivos, e incluye tanto a personas con marcas de expresión como a aquellas que aún no las presentan, volviéndose así un trabajo terapéutico y preventivo (Mattia et al., 2008). 
Esta área de actuación propone una nueva interacción con otras disciplinas, por ejemplo la dermatología, evidenciando que el trabajo en conjunto puede prevenir y disminuir la presencia de arrugas y lesiones en la piel, mediante una intervención natural y no invasiva como es el reequilibrio de las funciones estomatognáticas y la relajación de la musculatura facial (Franco y Scattone, 2002).

Actualmente, en Chile la fonoaudiología y su relación con la estética facial es un campo prácticamente no abordado por los fonoaudiólogos nacionales. Sin embargo, con el desarrollo de la profesión y la apertura de nuevas áreas de atención, la estética facial se ha convertido en un tema que cada día cobra relevancia y que requiere ser difundido. Por tales motivos, el propósito de este artículo es contribuir a la actualización de los conocimientos del fonoaudiólogo, particularmente en estética facial y atención primaria, además de ofrecer herramientas que aporten al desarrollo de esta área en Chile. Para ello se describirá un programa de estética facial en la atención primaria de salud (APS) en la ciudad de Sete Lagoas, Minas Gerais, Brasil.

\section{Desarrollo}

El envejecimiento es un proceso biológico y natural al que nos enfrentamos desde que nacemos y que varía de acuerdo con la herencia y predisposición genéticas, las características intrínsecas de cada individuo y la acción de múltiples factores externos (Teixeira y Guariento, 2010). Algunos de estos factores corresponden a exposición a la luz solar, consumo de cigarrillos y alcohol, agentes medioambientales (frío, sequedad, viento, contaminación, etc.), agentes metabólicos (carencia de vitaminas $A, C, E$ y ácido fólico), alimentación inadecuada, exceso de azúcar, baja hidratación, estilo de vida (falta de sueño, situaciones de estrés, etc.), presencia de radicales libres, factores mecánicos (por ejemplo, contracción repetitiva de los músculos faciales), entre otros (Franco, 2009).

Para comprender mejor el proceso es importante señalar que existen dos tipos de envejecimiento, uno intrínseco y otro extrínseco. El primero de ellos se refiere a la velocidad de envejecimiento que ocurre con el paso del tiempo, mientras que el segundo, corresponde al envejecimiento intrínseco sumado a causas externas (por ejemplo, la exposición a la luz solar, contaminación del aire, productos agresivos, tratamientos inadecuados, etc.). Para agravar aún más el daño extrínseco, el ritmo de autorreparación del organismo después de la exposición a agentes nocivos disminuye con la edad (Franco, 2009; Teixeira y Guariento, 2010). Así, a partir de los 26 años, las reacciones del organismo para combatir los radicales libres se vuelven más lentas, siendo necesario ayudarlo, por ejemplo, a través de la alimentación (rica en nutrientes antioxidantes, vitaminas, aminoácidos y sustancias naturales) y el estilo de vida (evitando el sedentarismo, la obesidad y el estrés) (Franco, 2009).

El rostro corresponde a la región más expuesta del cuerpo humano, por lo tanto, recibe en forma directa las agresiones del medio ambiente, además de ser una zona conformada por múltiples músculos que favorecen la aparición precoz de arrugas (Franco, 2009; Souza, 2012). Los músculos de la expresión facial son los más delicados y frágiles del 
cuerpo humano, se insertan bajo la piel y sus funciones movilizan el cutis, produciendo depresiones -en forma de línea o fosaperpendiculares a la dirección de las fibras de los músculos. La repetición de esos movimientos, día a día, durante la exteriorización de los sentimientos y la consecuente alteración de la fisionomía, acaba por marcar la piel y favorecer la aparición de arrugas (ver Tabla 1). Un hecho similar ocurre también al realizar las funciones de masticación, deglución, articulación y expresión facial durante la comunicación (Franco, 2009). Es posible, entonces, afirmar que en muchas ocasiones las personas son responsables de las arrugas que presentan en su rostro y, por lo tanto, los cuidados enfocados en esta zona deberían iniciarse en forma precoz.

Tabla 1

Edad de aparición de las arrugas

\begin{tabular}{|c|c|}
\hline $\begin{array}{l}20-25 \\
\text { años }\end{array}$ & $\begin{array}{l}\text { Líneas orbiculares visualizadas con la } \\
\text { expresión facial. }\end{array}$ \\
\hline $\begin{array}{l}\text { 40-45 } \\
\text { años }\end{array}$ & $\begin{array}{l}\text { Arrugas periorbiculares, frontales y glabelares. } \\
\text { Acentuación del surco nasogeniano, pliegues } \\
\text { transversales en la región del cuello. } \\
\text { Formación de bolsas de grasa en la región } \\
\text { palpebral. }\end{array}$ \\
\hline > 55 años & $\begin{array}{l}\text { Acentuación de las arrugas y pliegues, } \\
\text { atenuación de la eminencia malar, depresión } \\
\text { del surco nasolabial y ptosis facial. }\end{array}$ \\
\hline
\end{tabular}

Las arrugas pueden ser clasificadas en tres categorías: pliegues 0 arrugas gravitacionales (ptosis); arrugas finas; y arrugas de expresión o dinámicas (consecuencia de movimientos repetidos de los músculos faciales y que pueden variar su distribución e intensidad según factores intrínsecos y extrínsecos del envejecimiento). No se incluyen las arrugas estáticas que aparecen en ausencia de movimiento y pueden ser interpretadas como fatiga de las estructuras que constituyen la piel.

Entre las señales que manifiestan el envejecimiento en el rostro es posible mencionar las arrugas periorbiculares, acumulación o exceso de piel palpebral, bolsa de grasa bajo los ojos, acentuación del surco nasogeniano, además de las arrugas glabelares y frontales. La flacidez puede estar presente, junto con una acumulación o exceso de piel y grasa en la región mandibular ("papada"). Esas señales pueden aparecer todas, en conjunto o separadamente y en menor o mayor intensidad, dependiendo de cada individuo y de la contribución de factores externos (Toledo, 2006).

Como se mencionó anteriormente, se cree que la aparición de las arrugas puede estar relacionada con alteraciones miofuncionales implicando muchas veces disfunciones del sistema estomatognático. Así, la fonoaudiología ha dirigido su atención clínica hacia la motricidad orofacial -específicamente la estética facial- con el fin de proporcionar al individuo una apariencia joven, saludable, con expresiones suaves, amenizando los efectos del envejecimiento observados en el rostro y, consecuentemente, en el funcionamiento de todo el complejo orofacial (Souza et al., 2005).

Finalmente, es importante destacar que la acción de la estética facial no debe considerarse como una terapia individualizada en la búsqueda del rejuvenecimiento facial, sino que por el contrario, su acción puede contribuir y complementar otros tratamientos faciales sean ellos dermatológicos, plásticos o estéticos, destacando que cada uno de estos procedimientos tiene objetivos y planes terapéuticos específicos, pero todos están orientados a una meta en común (Souza et al., 2005). 


\section{Atención Primaria en Salud y Núcleo de Apoyo a la Salud de la Familia}

La Atención Primaria en Salud (APS) representa un conjunto de conocimientos y procedimientos que busca una amplia intervención en diversos ámbitos de la salud, con el fin de producir un efecto positivo en la calidad de vida de la población, además de ser el primer contacto en la red asistencial del sistema de salud. En este sentido, la Estrategia de Salud de la Familia (ESF), vertiente brasilera de la APS, se identifica como la puerta de entrada a un sistema de salud constitucionalmente fundado en el derecho a la salud y en la equidad del cuidado, además de eso, jerarquizado y regionalizado, como es el caso del Sistema Único de Salud (SUS) (Silva et al., 2012).

En el año 2008, el Ministerio de Salud de Brasil creó los Núcleos de Apoyo a la Salud de la Familia (NASF) para apoyar la inserción de la ESF en la red de servicio y ampliar la cobertura, capacidad de resolución, territorialización, regionalización, así como la ampliación de las acciones de la APS. Un NASF debe estar conformado por un equipo en el cual los profesionales de diferentes áreas de conocimiento actúan en conjunto con los profesionales de los equipos de salud de la familia, compartiendo y apoyando las prácticas de salud en terreno, bajo la responsabilidad de los equipos de salud de la familia. Es así que los equipos de NASF pueden estar compuestos por profesionales de diversos ámbitos: asistentes sociales, kinesiólogos, fonoaudiólogos, nutricionistas, psicólogos, terapeutas ocupacionales y profesores de educación física, entre muchos otros. Este organismo actúa considerando algunas directrices como acción interdisciplinar e intersectorial, educación en salud permanente para los profesionales y la población, desarrollo de noción territorial, integración, participación social, educación popular, promoción de la salud y humanización (Ministério da Saúde, 2008).

EI NASF está compuesto por nueve áreas estratégicas: salud del niño y adolescente, salud mental, rehabilitación y salud integral del anciano, alimentación y nutrición, servicio social, salud de la mujer, asistencia farmacéutica, actividades físicas y prácticas corporales (donde se inserta la fonoaudiología estética) y prácticas integrativas y complementarias (Ministério da Saúde, 2008). Los procesos de trabajo en el NASF deben ser estructurados, priorizando la atención compartida e interdisciplinaria. Existe intercambio de conocimientos, capacitación y responsabilidades compartidas, generando experiencia para todos los profesionales involucrados mediante amplias metodologías, tales como estudio y discusión de casos y situaciones, proyectos terapéuticos, orientaciones y atención conjunta, etc. Las intervenciones directas del NASF frente a los usuarios y familias son realizadas siempre en compañía de los equipos de salud familiar con discusiones y negociación a priori entre los profesionales responsables, según sea el caso (Ministério da Saúde, 2008).

Actualmente en la ciudad de Sete Lagoas, Minas Gerais, Brasil existen cinco equipos de NASF que actúan en conjunto con las ESF, compartiendo las prácticas en salud en los territorios correspondientes en los cuales cada NASF está inscrito (Ministério da Saúde, 2008). 


\section{Programa de fonoaudiología estética en Sete Lagoas, Minas Gerais, Brasil}

El programa de fonoaudiología estética realizado en la ciudad de Sete Lagoas, Minas Gerais, Brasil, se enmarca en el contexto de atención primaria, específicamente en los NASF.

En los NASF el fonoaudiólogo, en conjunto con los otros profesionales del equipo, busca fomentar la integración del cuidado físico y mental de los usuarios por medio de la calificación y complementariedad del trabajo interdisciplinario (Ministério da Saúde, 2009). Las actividades aquí desempeñadas por el fonoaudiólogo son diversas, destacando principalmente la realización de actividades grupales.

Entre las áreas estratégicas del NASF, el conjunto conocido como "prácticas corporales" (PC) estimula la interacción mente-cuerpo, proporcionando a los participantes una mayor conciencia de su integridad como ser humano, favoreciendo la mejoría de la calidad de salud y de vida. Actúa en la promoción y prevención de la salud, ayuda en el tratamiento de enfermedades y contribuye a la humanización de los servicios de salud (Ministério da Saúde, 2009). El trabajo es realizado dos veces por semana en un grupo permanente, con gran rotación de participantes y tiene como principales responsables a técnicos profesionales de fisioterapia (kinesiología) y educación física.

Considerando la evidencia acerca de los beneficios de la fonoaudiología estética y sumado a los objetivos que propone el grupo de PC dentro del NASF, desde junio del año 2014 se incluyó en el grupo de PC-NASF de Sete Lagoas la práctica de fonoaudiología estética, a cargo de la fonoaudióloga
Taysa Silva. El trabajo fonoaudiológico se efectúa quincenalmente en encuentros grupales, donde los participantes -guiados por el fonoaudiólogorealizan ejercicios de relajación y perfeccionamiento muscular durante 30 minutos. En cada encuentro participan cerca de 40 personas, con un total de 160 personas en 12 meses de puesta en marcha del programa, predominando las mujeres, adultas y adultas mayores. Las necesidades de cuidado más frecuentes referidas por los participantes son los ejercicios faciales relacionados con la mímica facial.

Es importante aclarar que en la práctica de fonoaudiología estética no se utiliza u orienta acerca del uso de cosméticos, y la intervención en la piel y musculatura ocurre de manera directa por la realización de masajes y ejercicios.

La sesión en general se estructura de la siguiente forma: inicialmente, en los primeros 5-10 minutos, se realizan técnicas de relajación utilizando música suave y apropiada para lograr la relajación mental. Posteriormente se realiza relajación de toda la musculatura corporal, enfocada en la musculatura orofacial, cuello, hombros, abdomen y miembros superiores e inferiores.

El calentamiento de la musculatura facial tiene una duración aproximada de 10 minutos y se desarrolla mediante una serie de movimientos de manipulación y soltura facial, los cuales son autoaplicados por cada participante en la musculatura de todo el rostro. Luego del proceso de estiramiento muscular, la musculatura de la mímica facial es trabajada en forma específica. Se utilizan diferentes ejercicios: los isocinéticos, que proporcionan una dinámica muscular facial y contribuyen a la elevación del tono muscular; los 
isométricos, que desarrollan resistencia sobre la base de la tensión muscular; y los isotónicos, que proporcionan un trabajo muscular dinámico y el alargamiento y acortamiento del músculo (Tasca, 2004).

En este paso, se informa a los usuarios que los ejercicios deben ser ejecutados en forma pausada y con cautela. Corresponde a la etapa más relevante de la sesión y en ella se trabaja con ejercicios específicos para la musculatura de la frente, nariz, ojos, mejillas, boca, mentón, maxilar, papada y cuello (ver Tabla 2). El objetivo propuesto es mejorar la movilidad muscular, aumentar la fuerza, disminuir y elevar el tono muscular, además de propiciar una mejor circulación sanguínea. Es importante destacar que para lograr la relajación facial y conseguir un resultado efectivo se deben complementar los ejercicios con relajación y estiramiento facial.

La sesión finaliza cuando los usuarios han completado la serie de ejercicios propuestos. En esta instancia, se les entregan las instrucciones para que repliquen los ejercicios en su hogar, los cuales deben ser practicados como mínimo tres veces por semana para evidenciar resultados.

Table 2

Ejercicios musculares utilizados en el programa de Estética Facial-NASF

\begin{tabular}{|c|c|}
\hline Músculo ejercitado & Descripción \\
\hline Frontal & - $\quad$ Elevar las cejas \\
\hline Nasal y del ala de la nariz & - $\quad$ Abrir y cerrar las narinas \\
\hline Orbicular del ojo y palpebral & $\begin{array}{l}\text { - } \quad \text { Abrir bien los ojos } \\
\text { - } \quad \text { Pestañear varias veces } \\
\text { - } \quad \text { Cerrar los ojos y mantenerlos cerrados contrayendo fuerte } \\
\text { - } \quad \text { Mirar a los cuatro puntos cardinales sin mover la cabeza (el terapeuta puede ayudar } \\
\text { - } \quad \text { con su dedo) } \\
\text { Mirar la punta de la nariz }\end{array}$ \\
\hline Buccinador y cigomático & $\begin{array}{ll}\text { - } & \text { Sonreír sin mostrar los dientes } \\
\text { - } & \text { Sonreír mostrando los dientes } \\
\text { - } & \text { Succionar las mejillas } \\
\text { - } & \text { Inflar mejillas } \\
\text { - } & \text { Tirar beso y sonreír mostrando dientes (beso-sonrisa) } \\
\end{array}$ \\
\hline Orbicular de los labios & $\begin{array}{ll}\text { - } & \text { Protruir labios } \\
\text { - } & \text { Protruir labios, lateralizando } \\
\text { - } & \text { Tirar beso } \\
\text { - } & \text { Tirar beso y sonreír mostrando dientes (beso-sonrisa) } \\
\text { - } & \text { Labios abiertos en forma de “ } O \text { ", juntando } \\
\text { - } & \text { Labios abiertos en forma de “ } O \text { ", lateralizando } \\
\text { - } \quad \text { Labios abiertos en forma de “ } O \text { ", juntando y lateralizando }\end{array}$ \\
\hline Mentoniano y masetero & $\begin{array}{ll}\text { - } & \text { Dientes ocluidos, morder con fuerza* } \\
\text { - } & \text { Labios unidos, proyectar mandíbula hacia anterior y hacia lateral* } \\
\text { - } & \text { Abrir la boca lentamente, proyectando la mandíbula hacia lateral* }\end{array}$ \\
\hline Suprahioideos y platisma & $\begin{array}{ll}\text { - } & \text { Empujar la lengua contra papila retroincisiva } \\
\text { - } & \text { Empujar la lengua contra el paladar duro } \\
\text { - } & \text { Inclinar cabeza hacia atrás, abrir y cerrar la boca** } \\
\text { - } & \text { Inclinar cabeza hacia atrás, abrir y cerrar la boca, sacando y entrando la lengua** }\end{array}$ \\
\hline
\end{tabular}

Nota: Es importante considerar las diferencias individuales propias de cada individuo, por lo cual, se deben adaptar algunos movimientos.

*Pesquisar disfunción de ATM antes de ejecutar ejercicio y adaptar según condiciones referidas por los usuarios.

**Estar atento a posibles alteraciones cervicales de la persona. Evitar ejercicio en estos casos. 


\section{Discusión y comentarios finales}

Como se mencionó previamente, la estética facial es un área relativamente nueva en el ámbito fonoaudiológico. Por tal motivo no es extraño que muchos fonoaudiólogos no posean suficiente formación respecto de la intervención realizada en este campo. Así lo demuestra un estudio efectuado en Brasil en el cual se consultó a 22 fonoaudiólogos especialistas en $\mathrm{MO}$, de los cuales sobre el $80 \%$ nunca había intervenido en estética facial y sus conocimientos en torno a la temática eran limitados, careciendo de formación más específica (Souza et al., 2005). Respecto de este tema, en el medio nacional no existen estudios, por ende no es posible evidenciar el nivel de conocimiento de los profesionales fonoaudiólogos acerca de estética facial. Sin embargo, es factible deducir que los porcentajes de desconocimiento del tema en Chile pueden ser mayores que los documentados en Brasil, donde esta área está mucho más desarrollada.

Si bien el objetivo del presente artículo no era evidenciar los resultados del programa de fonoaudiología estética aplicado, al consultar a la población atendida, los usuarios manifestaron percibir cambios en los patrones de deglución, masticación y respiración. También señalaron percibir cambios en su musculatura facial, con atenuación y suavización de las arrugas y líneas de expresión. Al contrastar la información referida por los participantes del programa con lo establecido en la evidencia, existe clara concordancia. Diversos estudios realizados en el área de estética facial (Frazão y Manzi, 2012; Silva, Vieira y Motta, 2009; Souza, 2012; Souza, Guerra, Barbosa y Porto, 2013) muestran que la intervención fonoaudiológica mejora la percepción de cara flácida y rostro envejecido, favorece la relajación facial, disminuye las líneas de expresión y arrugas, mejora las funciones estomatognáticas, mejora la tonicidad facial, incrementa la sensación de bienestar corporal y autosatisfacción, entre otras. Pese a ello, es importante considerar que la descripción del programa y su aplicación no garantizan su validez.

Un punto relevante de comentar es la modalidad de la intervención que puede ser individual, grupal o mixta. Según lo establecido en la literatura, la forma de trabajo individual propone ejercicios como estiramiento, masajes, modificaciones postulares, reequilibrio de las funciones estomatognáticas, eliminación o disminución de los movimientos compensatorios, ejercicios musculares, etc. (Souza, 2012). La modalidad de intervención expuesta anteriormente fue grupal y varía significativamente con respecto a la individual. El trabajo en grupo dificulta la consideración de las variables propias de cada sujeto, enfocándose de esta forma en los ejercicios generales y específicos que puedan favorecer a los participantes. Sin embargo, la intervención grupal aporta ventajas como el aumento de la motivación, la socialización de las experiencias y de lo aprendido, además de fortalecer los vínculos y el mutuo apoyo que se puede dar entre los participantes respecto de la autoimagen facial (Paes, Toledo y Silva, 2007).

En cuanto a la duración del programa no existe un claro consenso, pudiendo variar entre 5, 10, 12 sesiones (tres meses) e incluso 20 sesiones (cinco meses). Existe acuerdo, sin embargo, en que debería realizarse como mínimo una sesión por semana. Como en toda terapia fonoaudiológica, el éxito de la 
intervención se ve supeditado a factores externos, entre los que destacan: el nivel de compromiso y participación del usuario (es muy importante la asistencia a las sesiones y la realización de los ejercicios en el hogar, idealmente con una frecuencia diaria) (Paes et al., 2007), el nivel de autoconciencia/propiocepción respecto de las dificultades, y los cambios en el estilo de vida (por ejemplo exposición al sol, consumo de cigarrillo, etc.), entre otros.

De esta forma el rol del fonoaudiólogo en estética facial no solo se centra en realizar un entrenamiento muscular propiamente tal, sino que además debe informar al usuario acerca de las bases anatómicas que fundamentan el trabajo, indagar en los factores asociados al motivo de consulta, orientarlo sobre el trabajo con estética facial e indagar en el impacto emocional que pueda generar esta problemática. Todo lo anterior con el objetivo de realizar una intervención integral y efectiva, que permita lograr la conciencia y vinculación del usuario con la terapia. Por otra parte, es importante destacar que el trabajo de la fonoaudiología estética no solo debe relacionarse con el beneficio del rejuvenecimiento, sino que también es posible que se beneficien otros tipos de usuario, por ejemplo, personas con quemaduras de cabeza y cuello que presenten secuelas estéticas. La intervención desde la motricidad orofacial, específicamente de la estética facial, puede ser un aporte sustancial en este y otro tipo de usuarios.

El propósito de este artículo es contribuir en la actualización de los conocimientos del fonoaudiólogo, particularmente en estética facial y atención primaria, además de aportar con herramientas que permitan el futuro desarrollo de esta área en Chile. Al respecto, en Brasil existe mayor experiencia sobre dichos temas y ello favorece el desarrollo de la profesión, facilitando además el trabajo del fonoaudiólogo. Por tales motivos, es recomendable abrir nuevos campos en nuestro medio que permitan la inclusión del profesional fonoaudiólogo en otras áreas de intervención, desarrollar programas de promoción en salud que aporten con mayor conocimiento acerca del rol profesional a nivel nacional, fomentar el trabajo multidisciplinario y mancomunado con otras áreas (en este caso dermatólogos, cirujanos plásticos, kinesiólogos, etc.) e idealmente, incorporar la formación básica en estética facial en los programas de pregrado. 


\section{Referencias}

Comitê de Motricidade Orofacial. (2004). Motricidade orofacial: como atuam os especialistas. São Paulo: Pulso Editorial.

Conselho Federal de Fonoaudiologia. (2008). Resolução CFFa № 352. Jornal do CFFa, 9(37), 6-8.

Franco, M. Z. y Scattone, L. (2002). Fonoaudiologia e dermatologia um trabalho conjunto e pioneiro na suavização das rugas de expressão facial. Fono Atual, 5(22), 60-66.

Franco, M. Z. (2004). Fonoaudiologia e estética facial: um novo alcance da motricidade oral. En Comitê de Motricidade Orofacial - SBFa (Ed.), Motricidade orofacial: como atuam os especialistas (pp. 289-295). São José dos Campos: Pulso Editorial.

Franco, M. Z. (2009). A fonoaudiologia que rejuvenesce. São Paulo: LivroPronto.

Frazão, Y. y Manzi, S. B. (2012). Eficácia da intervenção fonoaudiológica para atenuar o envelhecimento facial. Revista CEFAC, 14(4), 755-762.

Mattia, F., Czlusniak, G., y Ricci, C. (2008). Contribuição da fonoaudiologia na estética facial: relato de caso. Revista Salus-Guarapuava, 2(2), 15-22.

Ministério da Saúde, Brasil. (2008). Portaria GM № 154 de 24 de Janeiro de 2008 - Cria os Núcleos de Apoio à Saúde da Família - NASF. Diário Oficial da União Seção 43.

Ministério da Saúde, Brasil. (2009). Diretrizes do NASF: Núcleo de Apoio à Saúde da Família. Cadernos de Atenção Básica, 27. Brasília: Autor.
Paes, C., Toledo, P. N., y Silva, H. (2007). Fonoaudiologia e estética: estudo de casos. Revista CEFAC, 9(2), 213-220.

Silva, A. T. C. et al. (2012). Núcleos de apoio à saúde da família: desafios e potencialidades. Cad. Saúde Pública, 28(11), 2076-2084.

Silva, N. L., Vieira, V. S., y Motta, A. R. (2009). Eficácia de duas técnicas fonoaudiológicas da estética facial no músculo orbicular dos olhos: estudo piloto. Revista CEFAC, 12(4), 102-109.

Souza, C. (2012). Intervenção miofuncional estética: uma nova proposta para o rejuvenescimento facial. Fragmentos de cultura, 22(1), 73-79.

Souza, C., Guerra, J., Barbosa, M. A., y Porto C. (2013). Rejuvenescimento facial por intervenção miofuncional estética. Revisão integrativa. Medicina Cutánea IberoLatino-Americana, 41(4), 165-171.

Souza, E., Morais, W., Silva, H., y Cunha, D. (2005). O conhecimento do fonoaudiólogo especialista em motricidade orofacial sobre atuação em estética facial. Revista CEFAC, 7(3), 348-355.

Tasca, S. T. (2004). Programa de aprimoramento muscular em fonoaudiologia estética Facial (PAMFEF). São Paulo: ProFono.

Teixeira, I. y Guariento, M. (2010). Biologia do envelhecimento: teorias, mecanismos e perspectivas. Ciência \& Saúde Coletiva, 15(6), 2845-2857.

Toledo, P. N. (2006). Fonoaudiologia e estética: a motricidade orofacial aplicada na estética da face. São Paulo: Lovise. 